\title{
Queerizando las Facultades de Educación: una escuela inclusiva es posible
}

\author{
Queering Education Faculties: an inclusive school is possible \\ Queeritzant les Facultats d'Educació: una escola inclusiva és \\ possible
}

\author{
Guillermo Soler-Quílez. Universitat d'Alacant, España. \\ guillermo.soler@gcloud.ua.es \\ http://orcid.org/0000-0002-3535-3004
}

\section{Extended abstract}

This paper presents research that revolves around queer pedagogical methodologies, especially regarding language and literature teaching. It addresses the engagement with queer teaching methods implemented by Education Faculties both in the Anglo-Saxon world and in the Spanish context.

Following the premise that literature is able to transmit particular values, these analyses have experimented with proposals that include the use of LGBTQ literature, especially picture books. Using a qualitative design, the purpose of the study was to introduce children's literature that portrays LGBTQ characters and families to explore the perceptions of future teachers in the elementary classroom regarding queer culture, and the possibility of reading these picture books as instruments to interrupt heteronormativity and to tackle homophobia. It should be noted that this kind of interruption at the elementary level is often avoided, as it addresses sexual identity and it could be considered by some to be inappropriate for the elementary curriculum. The reality, however, is that sexual identity is included in early classrooms as heterosexuality: a single story of family and sexuality. Given the absence of inclusive and diversity practices that should have accompanied social policies, schools have in fact perpetuated the hostile and homophobic territory experienced by people who do not meet gender or sexuality expectations. Subsequently, a new model of queer pedagogy has emerged, demanding new proposals from schools at all levels: from curricular organisation to the types of readings pupils have access to.

Preservice teachers' personal beliefs appear to influence their avoidance of gender and sexuality topics in the classroom. These beliefs are often shaped by their own school and life experiences, personal beliefs, and societal attitudes. In the training of future teachers in the United States, the inclusion of affective-sexual orientation issues begins to emerge in the university plans for their preparation at the beginning of the new millennium. After the descriptive analysis of teacher training programs carried out by Todd Jennings and Gary Sherwin (2008), the results indicate that only $55.6 \%$ of the programs include these topics within the official curriculum and, when they appear, the topics remain isolated, with little support material and no professional practice. In their conclusions, they suggest that Primary Education Faculties programs can work to sustain homophobic and 
heterosexist school cultures through the omission of affective-sexual orientation issues, despite the apparent commitment to prepare teachers in this sense. In some states of the USA some teachers have been fired because of controversies stemmed from introducing children's literature with LGBTQ themes in the classroom. These situations illustrate the potential of complicated school environments and present need for conversations in Primary Education Faculties in order to broach how and why teachers might select texts with controversial themes for classroom instruction.

Some teachers applied picture books including LGTBQ characters to build classrooms that were more respectful towards difference. They started with a simple premise: introducing the fact that samesex people can love each other makes it possible to destabilise both heteronormative family notions, and the presumption that heteronormativity constitutes the organising principle of every society. Queer pedagogy through different investigations carried out in schools of education around the world (Blackburn and Carn, 2011; Cumming-Potvin and Martino, 2014; Kelly, 2015 Martino and Cumming-Potvin, 2011, 2014, 2016; Paiz, 2019, Steck , and Perry, 2017) presents some common points of view. First: the student body welcomes reading proposals that refer to sexualities or gender issues. Second: the faculty displays a greater degree of rejection towards these proposals. Third: some proposals indicate that the inclusion of readings in the daily practice does not seem sufficient, that additional intervention is necessary. In the Spanish academic environment in the last decade, different work has appeared on affective-sexual and gender diversity and education. Melani Penna Tosso (2012) completed a PhD where the absence of concrete aspects on the questions of sexualities and genders in teacher training is portrayed. In 2016, Stefano Barozzi published a thesis in which he presented an experience with teachers who take training courses at the university to expand their knowledge of LGBTQ issues. From the perspective of students, Ramírez's (2012) thesis has focused on addressing adolescent voices that deviate from the norm and Renovell (2017) analyses the attitudes of secondary school students towards affective-sexual and gender diversity.

In one of the most recent investigations, Buchanan, Tsichida, Bellows and Shear (2019) used picture books displaying family diversity with future primary school teachers. In initial surveys, the participants showed an open predisposition to include LGTBQ families in their teaching practice. However, there was a change in attitude when the preservice teachers began to work directly with texts. Through the discussion groups and the final written responses, it was observed that a large majority was against including these albums in their classes. They outlined three main arguments: religious beliefs, personal beliefs and, finally, a fear of conflict.

The sample of the research presented in this article included students of the subject Didactics of Reading and Writing, within the Education Faculty of Alicante University during the fall semester (between September and October, 2018). A different text was read in the classroom per week, and this text would then be incorporated into discussion groups. Students analysed the texts' content, paying special attention to the representation of the LGBTQ community, but they also discussed the possible use of these texts in primary courses. The aim was firstly to critically assess and analyse illustrated albums with an LGTBQ presence and, secondly, to establish spaces for dialogue and discussion to raise awareness about the importance of working with these readings in Primary classrooms. Participants reported a range of positive responses to the texts presented. However, some of the participants anticipated conflict with their use in the classroom, due to religious beliefs or problems with families. A discussion of the findings also includes teachers' intentions to persevere in their future teaching activities related to LGBTQ topics by using children's literature. Language, sexuality and norms are recognized to be rooted in discourse and found in the daily life of primary school students, for this reason, this type of reading facilitates the questioning of the heteronormative model and allows to stop assuming that this model is the only valid one. The findings reflect the following: to build a school where all people feel included, respected and accepted, it is necessary to renew the readings in the classroom. One of the most innovative contributions around the debate on queer reading in class was the importance of having received 
critical training when choosing these readings, an issue which numerous participants highlighted and which was one of the main objectives of this research.

As teacher educators, our proposal offers a significant space for preservice teachers to begin thinking about the new materials they can select to teach social studies content. However, we recognize the pervasive problem of LGBTQ issues as being largely absent in teacher education courses (HermannWilmarth, 2010), especially at the elementary level and, specifically, in elementary social studies. If affective-sexual and gender diversity were explicitly included in the official curriculum from the first year of the degree in a transversal way -that is, if we queer the Faculty of Education-, we anticipate this rejection would decrease progressively in each course. We suggest transforming the priorities in the programs of the Faculties of Education to train faculty so that they are able to generate spaces where preservice teachers explore their beliefs, investigate teaching materials and understand the need to address controversial issues in the classroom. Course experiences like the ones described here are essential to creating elementary classroom spaces that recognize gender, affective or sexual differences and work to generate schools where our youth can recognize themselves and feel protected and safe. These objectives cannot be relocated to single class sessions or short activities in a separate course. The need to foster an environment in which teachers are recognized as allies of the LGTBQ community in public school classrooms cannot be ignored. The goal is to train the teachers of the future with tools to teach that diversity enriches all of us, it favours the lives of LGTBQ students and, in short, it contributes to building an inclusive school, a queer school.

Keywords: Queer pedagogy, LGBTQ, education, inclusive school

\section{Resumen}

El siguiente trabajo ofrece una revisión en torno a las prácticas pedagógicas especialmente en el área de lengua y literatura. Un recorrido sobre las propuestas llevadas a cabo en diferentes facultades de educación tanto en el ámbito anglosajón como en el contexto español. Bajo la premisa de que la literatura puede transmitir unos determinados valores, estas investigaciones han experimentado con propuestas de lecturas de temática LGTBQ. A continuación, muestra una investigación llevada a cabo en la Facultad de Educación de Alicante, en la asignatura de Didáctica de la Lectura y de la Escritura, cuyo propósito principal consistía en incluir estas lecturas diversas con el fin de concienciar a los y las futuras maestras de la importancia de abordar estos temas en el aula de primaria, valorando y analizando una serie de álbumes ilustrados y favoreciendo grupos de discusión en torno a estas obras y a su posible uso. En las conclusiones se puede observar cómo, a pesar de que todavía se manifiestan algunas voces en contra, la mayoría del alumnado muestra su receptividad a incluir en sus clases estos temas; además se muestra predispuesto a desarrollar un pensamiento crítico en torno a las obras y a la representación de la diversidad afectivo-sexual y de género en los textos.

Palabras clave: Pedagogía queer, LGTBQ, educación, escuela inclusiva

\section{Resum}

El següent treball ofereix una revisió al voltant de les pràctiques pedagògiques queer, especialment dins de l'àmbit de llengua i la literatura. Un recorregut sobre les propostes que s'han fet en diverses facultats d'educació tant en l'àmbit anglosaxó com en el context espanyol. Partint que la literatura pot transmetre uns valors determinants, aquestes investigacions han experimentat amb propostes de lectures de temàtica LGTBQ. A continuació, es mostra una investigació realitzada en la Facultat d'Educació d'Alacant, en l'assignatura de Didàctica de la Lectura i de l'Escriptura, amb el propòsit principal d'incloure aquestes lectures diverses amb la finalitat de conscienciar als i les futures mestres de la importància d'afrontar aquests temes a l'aula de Primària, valorant i analitzant una sèrie d'àlbums il-lustrats i afavorint grups de discussió en torn a les obres i un hipotètic us. Les 
conclusions assenyalen que, malgrat que encara es manifesten algunes veus en contra, la majoria de l'alumnat es mostra receptiu a incloure en la seua pràctica docent futura estos temes; a més, es mostra predisposat a desenvolupar un pensament crític al voltant de les obres i la representació de la diversitat afectiva, sexual i de gènere dels textos.

Paraules clau: Pedagogia queer, LGTBQ, educació, escola inclusiva

\section{Introducción}

A pesar de que vivimos en uno de los países más avanzados legalmente en los temas de derechos civiles para la gente LGTBQ, lo cierto es que estos avances legislativos no han ido acompañados de propuestas educativas que permitan ir educando a las próximas generaciones dentro de un marco de respeto e igualdad por las diferencias en torno a las sexualidades y los géneros. En las facultades de Educación resultan necesarias nuevas propuestas que permitan introducir en el aula temas con contenido LGTBQ. En este marco, desde el área de Lengua y literatura, se plantea la posibilidad de introducir textos que se alejen de la representación de la normatividad y del patriarcado, con el fin de propiciar en el alumnado la reflexión sobre el silencio en las escuelas ante este tema, así como facilitar el conocimiento de unos álbumes ilustrados que favorezcan el desarrollo de una práctica pedagógica queer. ¿A qué hace referencia este término anglosajón? La teoría queer traducida al castellano sin

En este marco, desde el área de Lengua y literatura, se plantea la posibilidad de introducir textos que se alejen de la representación de la normatividad y del patriarcado, con el fin de propiciar en el alumnado la reflexión sobre el silencio en las escuelas ante este tema, así como facilitar el conocimiento de unos álbumes ilustrados que favorezcan el desarrollo de una práctica pedagógica queer mucho éxito como torcida o marica, supone ejercer una mirada crítica en constante oposición a la normatividad: Queer frente a normal siempre, en palabras de una de sus más relevantes teóricas Judith Butler "normalizing the queer would be, after all, its sad finish" (1994, p.21). Una teoría que pretende demostrar la inexistencia de una sexualidad natural, cuestionando incluso el concepto de hombre y de mujer (Jagose, 1996). Por tanto, llevado a la pedagogía, o pedagogías en plural, como prefiere Mercedes Sánchez (2019), pretende enfrentarse a la dualidad normalidad/anormalidad, concienciar de las presiones que se ejercen sobre la diferencia en las aulas por no tener un determinado físico, por un color de 
piel, por cuestiones de clase y, sobre estas marginalidades aquellas personas que por cuestiones de género o de identidad sexual pueden incluso quedar doblemente rechazadas. Ese estigma denominado homofobia que implica el trato hostil generalizado, a nivel psicológico y social para aquellas personas que son percibidas como gais, lesbianas y bisexuales, también a aquellas que no mantienen el comportamiento que se espera a su sexo asignado por nacimiento (Bordillo, 2001).

Desde finales de los noventa, la mirada queer ha sido llevada a la educación, especialmente ha puesto su foco de atención en la formación de los docentes. Sin duda, uno de los lugares comunes para desarrollar esta nueva manera de entender la pedagogía ha sido desarrollada en las clases de lengua y literatura. Ya en 1997, Linda Eyre plantea a su alumnado de la Facultad de Educación una serie de lecturas críticas con el heterosexismo con el fin de empezar a concienciar a los futuros docentes de la importancia de este tema, unas lecturas adultas útiles para el alumnado universitario, no tanto para los niveles educativos inferiores. Centrándose en la formación del nuevo profesorado, entre 1993 y 1998, Arthur Lipkin (2002) impartió en la facultad de Educación de Harvard módulos para trabajar contenido gay y lésbico a través de textos. Por su parte, Janine Schall (Schall y Kauffmann, 2003) realizó una investigación con su alumnado, su trabajo consistía en realizar una búsqueda de información sobre la presencia de la diversidad afectivo-sexual y de género en las aulas, estas futuras y futuros docentes manifestaron dudas bien justificándose con la idea de que la infancia es una etapa demasiado temprana para hablar de educación, bien se excusaban en motivos religiosos para cuestionar estas prácticas...

Bien por el temor al conocimiento o bien por la falta de formación sobre cómo manejar las ideas, bien porque el currículum lo permite, el profesorado no produce acercamiento alguno a cuestiones LGTBQ en las aulas. Por tanto, se consolida un discurso heteronormativo a través prácticas escolares, tales como la selección de textos con un predominio absoluto de conductas heteronormativas, el planteamiento de un único modelo de familia, la enseñanza de la sexualidad con fines reproductivos, etc. (Meyer, 2007). Estas prácticas favorecen que las escuelas se conviertan en espacios que rechazan comportamientos o actitudes no normativas. "For homophobia in schools to lessen, teacher education programs must interrogate homophobia and the naturalization of heterosexuality. I have found the children's literature classroom to be a place where discussion of these issues can reach prospective elementary teachers" (Swartz, 2013, p. 52). Swartz (2003) plantea la opción de introducir las obras de literatura infantil de temática LGTB como ya se venía reclamando desde los primeros textos sobre pedagogía queer (Britzman, 1995; Jennings, 1994; Lynch, 2000; Sears, 1993; Sumara y 
Davis, 1998), además de la literatura, incluye el visionado del documental It's elementary talking about gay issues in school (Cohen y Chasnoff, 1997).

Bien por el temor al conocimiento o bien por la falta de formación sobre cómo manejar las ideas, bien porque el currículum lo permite, el profesorado no produce acercamiento alguno a cuestiones LGTBQ en las aulas. Por tanto, se consolida un discurso heteronormativo a través prácticas escolares, tales como la selección de textos con un predominio absoluto de conductas heteronormativas, el planteamiento de un único modelo de familia, la enseñanza de la sexualidad con fines reproductivos, etc.
Estudiantes y docentes en ciernes reflexionaron a través de la escritura sobre la falta de oportunidades en las otras clases de examinar aspectos controvertidos, y se sintieron bien al conocerse exploradores de nuevas maneras de vivir y trabajar en las aulas. No faltaron voces que favorecieron la discusión sobre conceptos difíciles en torno a la sexualidad y el género, así como alrededor de la cultura heteronormativa impuesta. Como parte de la discusión se habló también del sistema binarista y la naturalización de la heterosexualidad. Con el trabajo de los textos, se llegó a la conclusión de que las clases de literatura pueden ser el lugar ideal para luchar contra los prejuicios sociales, incluyendo el heterosexismo y la homofobia. Unos prejuicios que permiten según Spurlin (2000) dar forma al poder, construir vectores de dominación que

oscurecen, hasta hacer desparecer el deseo por el mismo sexo como eje significativo de la investigación pedagógica.

Denunciaba Rita M. Kissen (2002) que se han desarrollado programas de formación para maestros sobre racismo, sexismo, clasicismo, etc. en cambio, se ha trabajo muy poco en implementar e integrar la diversidad afectivo-sexual y de género en los planes de estudio en la formación docente. Cuando no cabe duda de cuán necesaria resulta la formación del profesorado en este sentido (Miller, 1999, Penna Tosso 2012). En 2003, Moje y MuQaribu, publican "Literacy and sexual identity" donde se posicionan claramente a favor de un currículum queer. Más allá de ver textos o autores LGTBQ, defienden las interconexiones entre sexualidad, identidad y literatura. En este sentido, Blackburn y Buckley (2005), Killoran y Pendlton Jiménez (2007) y Nieto y Bode (2012) apuestan por un currículum queer que asuma la sexualidad como necesidad primaria que acompañe a todo el conocimiento, especialmente en el área de Lengua y Literatura pues permite a las y los estudiantes experimentar la otredad, su propio otro identitario, como el otro social.

Straut y Sapon-Shevin (2002) señalan que el profesorado debe conocer, como mínimo, el significado de algunos términos básicos como lesbiana, gay, bisexual o trans. En realidad, tres 
ejes fundamentales deben ser atendidos si se pretende mejorar el sistema educativo: conocimiento, coraje y habilidades. El conocimiento con el que el profesorado debe contar consiste en manejar cierta información básica sobre el modo de vida de la gente LGTBQ en su propio entorno, sobre las familias homoparentales de su centro, así como cierta información sobre la importancia de la visibilidad del colectivo. Las y los docentes deben manifestar el coraje suficiente no solo para luchar contra la homofobia, sino también para responder ante posibles preguntas con claridad y naturalidad. Aunque parte del profesorado no se sienta cómodo integrando en el currículum contenidos LGTBQ puede, de igual modo, desarrollar las habilidades en su alumnado para ayudar a eliminar cualquier prejuicio que puedan albergar.

En la formación de los futuros docentes, en los planes universitarios para su preparación en Estados Unidos, empieza a aflorar la inclusión de temas de orientación afectivo-sexual a principios del nuevo milenio. Tras el análisis descriptivo realizado por Todd Jennings y Gary Sherwin (2008), los resultados señalan que tan solo el 55'6\% de los programas incluyen estos temas dentro del currículum oficial, sin embargo, aunque aparezcan quedan aislados, con escaso material de apoyo y nula práctica profesional. En sus conclusiones, sugieren que los programas de las facultades de Educación Primaria pueden trabajar para sostener las culturas escolares homofóbicas y heterosexistas a través de la omisión de temas de orientación afectivo-sexual, a pesar del aparente compromiso para preparar a los maestros y maestras en este sentido. Señala Mayo que: "teacher educators are not always prepared to understand LGBTQ issues or may resist lessons about homophobia or sexual and gender minority issues when they are part of preserve teacher education" (2013, p.71). Incluso la intención de hacer un lugar seguro en las escuelas puede ser problemático, pues puede llevar implícito que se considere un riesgo la sexualidad, las dificultades por las diferentes identidades e incluso los intercambios lúdicos que trabajan en los límites de la normalidad.

No es suficiente con leer textos con personajes LGTBQ, es necesario generar la reflexión entre el futuro profesorado con el fin de que pueda ampliar la propia visión de su mundo y extenderla de este modo en sus clases. Junto a Bills, Hermann-Wilmarth (2010) continúa desarrollando su labor de formadora de docentes introduciendo en su alumnado estos textos y sus discusiones. Cuando este entiende que se les pregunta por cómo sus creencias o asunciones pueden afectar a otras personas, esto les abre un camino hacia la autoreflexión que les puede permitir ampliar su percepción. Dentro de una asignatura sobre literatura infantil introdujo un tema con presencia gay y lésbica, debían realizar una reflexión en torno a la lectura y compartirla en grupos de discusión. Tras el análisis de estas reuniones, la autora llega a una conclusión: es necesario tiempo y espacio. Más tiempo para la propia reflexión y la compartida, así como más 
espacios de encuentro, de reflexión y de diálogo dado que todavía surgen voces entre sus estudiantes que muestran temor a trabajar con estos textos.

La pedagogía queer centrada en la formación docente continúa en el Oeste Australiano (Cumming-Potvin and Martino, 2014; Martino y CummingPotvin, 2011, 2014, 2016), en las pruebas de selección de maestras y maestros de Educación Primaria para empezar a trabajar se debía cumplir obligatoriamente con la elaboración de unidades didácticas para trabajar los diferentes modelos de familia utilizando textos literarios o virtuales como recursos pedagógicos. Para ello, a partir de una serie de álbumes ilustrados que incluían familias No es suficiente con leer textos con personajes LGTBQ, es necesario generar la reflexión entre el futuro profesorado con el fin de que pueda ampliar la propia visión de su mundo y extenderla de este modo en sus clases. homoparentales las y los docentes en ciernes debían diseñar prácticas concretas de aula. El estudio se amplió e incluyó la experiencia de unos pocos docentes que fueron abastecidos con recursos específicos y compartieron su experiencia en el aula, así como de un grupo de ocho profesores de Ontario, con experiencia en la práctica inclusiva en el aula sobre amor y parentesco entre personas no (hetero)normativas. Las narrativas de las y los docentes pueden servir como herramientas para construir y, a su vez, descubrir la manera en la que las creencias y valores del profesorado influyen en su práctica docente (en términos de autorizar o permitir unas lecturas sobre otras, por ejemplo). Asimismo, propicia la reflexión en torno a los prejuicios y presunciones que rigen el conocimiento parcial de la homosexualidad.

En definitiva, las diferentes investigaciones (Blackburn y Carn, 2011; Cumming-Potvin y Martino, 2014; Kelly, 2015 Martino y Cumming-Potvin, 2011, 2014, 2016; Paiz, 2019, Steck, y Perry, 2017) coinciden en algunas conclusiones. Primero: el alumnado recibe de buen grado propuestas de lecturas que se hacen referencia a las sexualidades o a las cuestiones de género. Segundo: el profesorado manifiesta mayor grado de rechazo a estas propuestas, bien por miedo a posibles conflictos con las familias, bien por conflictos internos. Tercero: algunas propuestas señalan que no parece suficiente con la inclusión de las lecturas en la práctica diaria, que es necesaria una intervención más profunda con el apoyo de las administraciones gubernamentales para que desarrollen políticas de reconocimiento que explique los límites impuestos por la heterosexualidad obligatoria y la negación de la legitimidad del deseo del mismo sexo; sin embargo, otras defienden que ya solo la inclusión de las lecturas ofrece resultados significativos (Hermann-Wilmarth y Bills, 2010; Martino, 2009; Paiz, 2018). 
En una de las más recientes investigaciones, Buchanan, Tsichida, Bellows y Shear (2019) utilizaron álbumes ilustrados donde se muestra la diversidad familiar con futuros docentes de primaria. En las encuestas iniciales mostraron una predisposición abierta a incluir a las familias LGTBQ en su práctica docente. No obstante, hubo un cambio de actitud cuando se empezó a trabajar directamente con los textos, a partir de los grupos de discusión y en las respuestas escritas finales se pudo observar que una amplia mayoría se posicionaba en contra de incluir estos álbumes en sus clases. Esbozaron tres argumentos principales: las creencias religiosas, las personales $\mathrm{y}$, finalmente, el miedo a generar conflictos.

En el ámbito académico español de la última década han aparecido diferentes trabajos sobre la diversidad afectivo-sexual y de género y la educación. Melani Penna Tosso (2012) realiza una tesis doctoral donde se retrata la ausencia de aspectos concretos sobre las cuestiones de sexualidades y géneros en la formación docente. En el año 2016, Stefano Barozzi publica una tesis en la que plantea una experiencia con docentes que realizan cursos de formación en la universidad para ampliar su formación en torno a cuestiones LGTBQ. De docentes a estudiantes, la tesis de Ramírez (2012) se ha centrado en las atender las voces adolescentes que se alejan de la norma y Renovell (2017) analiza las actitudes del alumnado en Secundaria hacia la diversidad afectivo-sexual y de género, mientras que otros estudios giran en torno a la influencia de la heternormatividad en la cultura (Carrera-Fernández, Cid-Fernández, Almedia, GonzálezFernández, Lameiras-Fernández, 2018).

Por su parte, desde la universidad de Cádiz, Begoña Sánchez-Torrejón (2017) denuncia la necesidad de queerizar la escuela a partir de los discursos del profesorado. En el ámbito autonómico, las universidades del Pais Valencià ofrecen cada vez más propuestas inclusivas: La universidad de Castellón Jaume I, cuenta con un proyecto de innovación educativa de la Facultad de Educación en la que Consol Aguilar Ródenas, además de sus publicaciones sobre la literatura infantil y juvenil de temática LGTB (2008) lleva a las aulas de los futuros docentes la diversidad afectivo-sexual y de género a través de la literatura infantil:

Nuestro propósito ha sido la implementación de la investigación proactiva de los y las estudiantes, como futuros maestros, cuestionando cómo sexualiza el sistema escolar desde la LIJ, y cómo se naturaliza el imaginario literario de la LIJ, para abordar los estereotipos que generan desigualdad y, así, poderlos combatir y transformar desde la igualdad, desde el compromiso, desde acciones educativas efectivas, ligadas a la inclusión de la diversidad sexual, a la defensa de los derechos humanos y a la formación de una ciudadanía crítica. Y podemos hacerlo desde el vínculo entre maestros, lectura y relaciones libres de violencia, incidiendo en la huella de la lectura 
y de nuestra biografía lectora, ligadas al desarrollo de la competencia literaria desde

la LIJ (Aguilar, 2017, p.7)

Desde València surgen propuestas como la de Oltra-Albiach y Pardo-Coy $(2016,2017)$ que inciden en la necesidad de incluir lecturas de temática LGTBQ en las aulas de Educación Infantil, partiendo de la formación de los y las docentes. Esta investigación se centra en el grado de Educación Primaria, en su último curso, a través de la asignatura de Didáctica de la Lengua y la Literatura. Durante el curso escolar 2018-9, la última sesión de la semana, se dedicó en parte a la lectura de un álbum ilustrado con representación diversa afectivo y sexualmente, así como de género con el fin de generar grupos de discusión entre el alumnado centrados en la necesidad de introducir la realidad LGTBQ en su práctica docente. Finalmente, en la última sesión se invitó al alumnado a reflexionar sobre la asignatura de manera general, así como la práctica docente, haciendo especial incidencia en las lecturas diversas.

La literatura puede ayudar a transformar el mundo, las lecturas que se realizan en la escuela deben ser elegidas con especial dedicación pues permiten transmitir y trabajar unos determinados valores, un pensamiento crítico (Llorens y Terol, 2015) que permitan desarrollar construir una ciudadanía consciente, competente y respetuosa con la diferencia. La capacidad empática de la persona lectora, la identificación con el personaje, facilita esta función de transmisión de valores a través de las emociones, miedos, conflictos... que despierta la literatura. Si en las facultades de educación, se lee y se trabaja con álbumes ilustrados LGTBQ se favorecerá un cambio de actitud en las y los futuros docentes cuya consecuencia se espera que sea un uso de este material en su futura práctica real.

El objetivo de esta investigación radica en la inclusión de lecturas LGTBQ en las clases de didáctica de lengua y literatura de la facultad de Educación, con el fin de concienciar a docentes en ciernes de la importancia de llevar la diversidad afectivo-sexual y de género a las aulas, así como de analizar sus posibles actitudes antes estos temas. Desde este planteamiento surgen los siguientes objetivos:

1. Valorar y analizar críticamente los álbumes ilustrados con presencia LGTBQ.

2. Establecer espacios de diálogo y discusión para concienciar sobre la importancia de trabajar con estas lecturas en las aulas de Primaria.

\section{Método}

2.1. Descripción del contexto y de los participantes 
En la Facultad de Educación de la Universitat d'Alacant, dentro del grado de Educación Primaria, se incluye la asignatura Didáctica de la Lengua y la Literatura en el último curso. Como contenidos principales de la asignatura destacan la literatura infantil y juvenil, el álbum ilustrado, el cómic así como sus posibilidades didácticas tanto para la formación de la competencia lecto-literaria en el alumnado, como su capacidad para introducir otros temas o valores en el aula. En el curso escolar 2018-19 en dos grupos de tarde se realizó la presente investigación, el primer grupo (grupo 7) formado por 41 estudiantes y el siguiente por 51 (grupo 8); un total de 92 estudiantes, 36 chicos y 56 chicas. Sin embargo, dado que se trata de grupos vespertinos, la asistencia a las clases queda reducida considerablemente, alcanzándose una media de 25 alumnas y alumnos por grupo y por clase, con edades dispares que van desde los 21 años hasta los 45 . El cuestionario final realizado el último día de clase fue contestado por un total de 15 chicas y 5 chicos en el grupo 7 y en el otro grupo, 13 chicas y 10 chicos.

\begin{tabular}{|l|l|l|l|l|l|l|}
\hline \multicolumn{2}{|l|}{} & & Frecuencia & Porcentaje & $\begin{array}{l}\text { Porcentaje } \\
\text { válido }\end{array}$ & $\begin{array}{l}\text { Porcentaje } \\
\text { acumulado }\end{array}$ \\
\hline Grupo 7 & Válido & Chicas & 15 & 75,0 & 75,0 & 750,0 \\
\hline & & Chicos & 5 & 25,0 & 25,0 & 100,0 \\
\hline & & Total & 20 & 100,0 & 100,0 & \\
\hline Grupo 8 & Válido & Chicas & 13 & 47,6 & 47,6 & 47,6 \\
\hline & & Chicos & 10 & 43,4 & 43,4 & 100,0 \\
\hline Total & & Total & 23 & 100,0 & 100,0 & \\
\hline estudiantes & Válido & Chicas & 28 & 65,1 & 65,1 & 65,1 \\
\hline & & Chicos & 15 & 34,9 & 34,9 & 100,0 \\
\hline & Total & 43 & 100,0 & 100,0 & \\
\hline
\end{tabular}

Tabla 1. Distribución del alumnado en el cuestionario final por Grupo y Género

Nota. La distribución por género entre grupos hace una suma total de 28 chicas y 15 chicos.

\subsection{Instrumentos}

Los instrumentos de recogida de información consistían en unos cuestionarios anónimos a final del curso, así como la observación directa del alumnado durante las sesiones dedicadas a la lectura de los álbumes de temática LGTBQ, poniendo el foco de atención en las actitudes y comentarios realizados durante y tras finalizar la lectura de los álbumes.

En cuanto a los álbumes ilustrados utilizados como elementos introductorios de las variables: lesbiana, gay, trans y homoparentalidad, pues se eligieron por mostrar la realidad diversa, pero 
también en función a unos criterios artísticos, literarios y visuales siguiendo las pautas de Temple, Martínez, Yokota y Naylor (2006) que destacan como elementos fundamentales la capacidad de desarrollar la empatía en el o la lectora, que no sea moralizante en exceso, que sea creíble, con argumento convincente y palabras escogidas y literarias, que permitan ver el mundo desde otro punto de vista. Rasgos que convierten a la literatura en un poderoso elemento para combatir la homofobia, pues descubriendo o incluso sintiendo el miedo o el rechazo que sufren las personas no normativas se puede empezar a destruir los prejuicios. Un aspecto fundamental que deben cumplir estas historias pasa por mostrar el rechazo social que vive el colectivo LGTBQ en su vida diaria pues solo retratando la realidad se podrá empezar a cambiar, a evolucionar (Soler-Quílez, 2019). Para mostrar la realidad lésbica, se leyó Titiritesa (Quintiá y Quarello, 2007), para la realidad gay, Rey y rey (De Haan y Nijland, 2004), las madres de sus protagonistas no aceptan de buen grado su diferencia; con Soy Jazz (Herthel, J., Jennings, J. y McNicholas, 2015) se mostraba la visibilidad trans que implica, en su caso, cierto rechazo de sus compañeros y compañeras de escuela; Aitor tiene dos mamás (Mendieta y Piérola, 2006) muestra una anécdota de intolerancia que permite trabajar también las familias homoparentales en su cotidianeidad.

\subsection{Procedimiento}

Entre los meses de septiembre y octubre, la asignatura de Didáctica de la Lengua y la Literatura se imparte de manera continua todos los días con dos horas de clase. En el transcurso habitual de las sesiones se leen diferentes textos con el fin de que el alumnado conozca de primera mano una selección de lecturas para llevar al aula cuando sean docentes en activo. En la última sesión de cada semana, se procedió a la lectura de álbumes ilustrados con presencia LGTBQ. Tras la lectura, junto al análisis de la obra tanto por su calidad literaria como artística, se propiciaba una reflexión en grupo en torno a la necesidad de introducir este tipo de lecturas en su práctica diaria como herramientas para luchar contra el machismo y la homofobia. Por último, la última sesión del curso: se pidió al alumnado que contestara de manera anónima un cuestionario en el que se preguntaba por aspectos generales de la asignatura, por la práctica docente recibida en general, y en particular por las cuestiones LGTBQ.

\section{Resultados}

En las diferentes sesiones dedicadas a la lectura de álbumes ilustrados no (hetero)normativos, el alumnado en general mostraba una valoración positiva de los álbumes, sin embargo, cuando se les preguntaba sobre su posible introducción en la práctica docente la actitud cambiaba en algunos y algunas de los estudiantes. Tres fueron los ejes en torno a los cuales se verbalizaba 
cierto grado de rechazo entre el alumnado: la familia, la edad y la religión. Las familias se

En las diferentes sesiones dedicadas a la lectura de álbumes ilustrados no (hetero)normativos, el alumnado en general mostraba una valoración positiva de los álbumes, sin embargo, cuando se les preguntaba sobre su posible introducción en la práctica docente la actitud cambiaba en algunos y algunas de los estudiantes. convertían en el primer escudo, en la primera excusa para no llevar este tipo de cuentos a la escuela, es decir, el alumnado temía que la inclusión de estos temas en el aula pudiera conllevar problemas de rechazo o incluso demandas a la inspección educativa por parte de las familias. Entre los dos grupos hasta un total de 9 estudiantes diferentes defendieron este posicionamiento a lo largo de las sesiones dedicadas a la lectura debate. La cuestión de la sexualidad también aparece como un problema, pues se defendía la posición de que el alumnado de Primaria todavía es demasiado pequeño para que aprendan este tipo de cuestiones, este argumento fue esgrimido en dos ocasiones por 4 estudiantes del grupo 7. La religión también aparece como un elemento perturbador, especialmente entre aquellas y aquellos estudiantes que se han formado en colegios religiosos y aseguraban no haber tenido ningún tipo de formación en este sentido y que tampoco la habían necesitado, entre ambos grupos 6 estudiantes manifestaron respuestas similares.

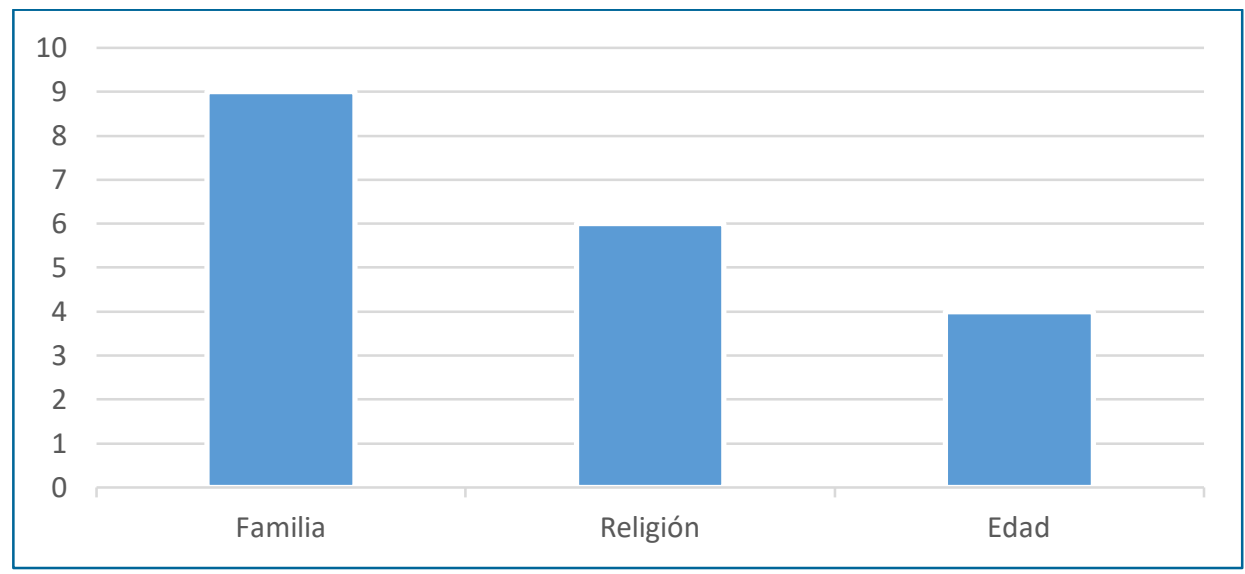

Gráfica 1. Ejes de rechazo a las lecturas inclusivas

Respecto a los resultados de los cuestionarios anónimos realizados a final de curso, se puede establecer dos posicionamientos respecto a la cuestión LGTBQ en ambos grupos. Del total de 43 estudiantes que contestaron el cuestionario final, un total de 13 escribieron respuestas que criticaban o cuestionaban la necesidad de incluir estos temas en las aulas de primaria, frente al 
30 restante que mostraba unas respuestas favorables ante la posibilidad de usar los álbumes ilustrados como herramienta para poder hablar de diversidad en el aula.

\begin{tabular}{|c|c|c|}
\hline Grupo total & & Porcentaje \\
\hline Favorables & 30 & $69,8 \%$ \\
\hline Desfavorables & 13 & $30,2 \%$ \\
\hline
\end{tabular}

Tabla 2. Actitudes favorables y desfavorables sobre lecturas diversas en el aula

Por un lado, voces que explicitan el rechazo a estos contenidos, justificando por diversos motivos, véase el siguiente ejemplo que se basa en la pérdida de tiempo: "se ha perdido mucho tiempo en estas discusiones/debates en clase". También se señala la necesidad de valorar otros temas, como el o la estudiante que reclamaba "variar los temas de LIJ" o algunos comentarios más en los que se acusaba al docente de haber planteado unas clases "algo radicales". Otra respuesta en esta dirección señalaba que el discurso del profesor resultaba "demasiado centrado en los aspectos de la homosexualidad, cuando hay otros aspectos más importantes para tratar". Como último testimonio destaca aquel que dice: "las clases se ha aprovechado para hablar siempre del feminismo o de la homosexualidad. Es un tema muy interesante, pero por desgracia los contenidos teóricos no tienen NADA que ver y son muy densos".

En sentido contrario, también se han producido respuestas positivas en las que se alaba la necesidad de romper los prejuicios en la escuela: "rompe la norma hablando con total normalidad de temas tabúes en la educación". También se destaca la oportunidad que han tenido de desarrollar su capacidad crítica: "enseñar a ser más críticos y traer y mostrar estos materiales tan útiles en el aula", del mismo modo en que han podido descubrir la manera de introducir en el aula estos textos, así como de seleccionar tales lecturas: "es interesante ver estos cuentos en clase y aprender cómo trabajarlos, cuales sirven y cuáles no". Por último, se pueden destacar algunas voces que ponen en evidencia la ausencia de estos temas en el devenir de su formación: "nunca nos habían dado la oportunidad de conocer la diversidad a través de los cuentos". Unas voces que señalan como aspecto fundamental en su formación "ampliar nuestra visión con el material LGTB y feminista" o "la concienciación con el colectivo LGTB debería ser generalizada en todo el grado"

\section{Discusión y conclusiones}

A pesar de que todas y todos los estudiantes del grado reconocieron haber presenciado en su etapa escolar algún tipo de actitudes homófobas, una parte de la clase se sorprendió de la 
propuesta de lectura. A través de esta práctica de pedagogía queer descubrieron que es

A través de esta práctica de pedagogía queer descubrieron que es importante construir un clima en el aula que permita a cada estudiante sentirse libre para expresarse o para actuar de un modo diferente sin que esto le convierta en objeto de burlas o de acoso importante construir un clima en el aula que permita a cada estudiante sentirse libre para expresarse o para actuar de un modo diferente sin que esto le convierta en objeto de burlas o de acoso. Como se observa en la tabla 2, hasta un 69,8\% del alumnado coincidía en que utilizar la literatura, especialmente los álbumes ilustrados leídos en las clases, les facilitaba la tarea de introducir en el aula la realidad LGTBQ. Es más, la mayoría tomó conciencia de la importancia de incluir en sus clases lecturas de esta temática para intentar reducir posibles situaciones de acoso escolar.

El segundo de los objetivos, que planteaba la necesidad de concienciar a las maestras y los maestros de las próximas generaciones sobre la importancia de trabajar la diversidad afectivosexual y de género en sus clases se ha cumplido. De hecho, algunas y algunos estudiantes se preguntaban sobre el motivo de la ausencia de estos temas en las diferentes asignaturas del grado, destacando la importancia que tendría una formación específica al respecto, un planteamiento que se ha reclamado desde hace ya varias décadas (Kissen, 2002; Miller, 1999; Penna Tosso, 2012; Straut y Sapon-Shevin, 2002). Sin embargo, la diversidad afectivo-sexual y de género y la escuela sigue siendo una de las tareas pendientes en el sistema educativo español en todos los niveles educativos incluyendo los grados de Educación. De ahí, que hasta un 30\%, mantenga todavía vigentes argumentos en contra de llevar la realidad LGTBQ a las aulas de Primaria como la edad o la religión, aspectos que ya señalaban Schall y Kauffmann, en el año 2003 y que coinciden en parte con los resultados de Buchanan, Tschida, Bellows, y Shear (2019). El temor a una respuesta negativa de las familias también aparece como un elemento clave para evitar el uso de estas obras. El resultado de esta visión puede redundar en evitar o censurar un determinado libro por tratarse de temas controvertidos para familias o estudiantes. Si bien, no mostrar familias diversas en las lecturas puede evitar conflictos con determinadas familias más tradicionales, también se está negando a las familias diversas su reconocimiento y existencia.

En el devenir de las clases, algunas voces coincidían con Hermann-Wilmart y Bills (2010), Martino, (2009) o Paiz (2018) en que no solamente es necesario introducir este tipo de lecturas, sino que también se requiere propiciar la reflexión y el debate sobre la discriminación y el acoso entorno a las personas no normativas. No se cuestiona que tanto el lenguaje como la sexualidad y las normas están arraigados en el discurso y en la vida cotidiana del alumnado de primaria, por 
este motivo, este tipo de lecturas facilita el cuestionamiento del modelo heteronormativo, permite dejar de asumir que este es el único válido. Si se quiere construir una escuela donde todas las personas se sientan incluidas, respetadas y aceptadas, es necesario renovar las lecturas en la escuela. Una de las aportaciones más innovadores en torno al debate sobre la lectura queer en las clases pasa por aquellas voces que destacaron la importancia de haber recibido cierta formación crítica a la hora de elegir estas lecturas, uno de los principales objetivos de esta investigación. Cierto es que no se da en el mercado editorial un número elevado de títulos con esta temática, no obstante, parte del alumnado tomó consciencia sobre la necesidad de ofrecer unas imágenes positivas sobre los personajes diversos o la importancia de que estas historias presenten un conflicto de carácter homófobo para mostrar la realidad sin edulcorarla, pues estas escenas facilitan que las y los lectores reflexionen sobre la vida cotidiana de las personas LGTBQ. También contribuye al proceso de empatía que implica la lectura y que permite ir derribando poco a poco ciertos prejuicios o estereotipos.

Si desde el primer curso del grado, de manera transversal, se incluyera la diversidad afectivosexual y de género en el currículum oficial de manera explícita, es decir, si queerizamos la

Debe ser una prioridad en los programas de las Facultades de Educación formar a sus docentes para que sean capaces de generar espacios donde aquellas personas todavía en formación exploren sus creencias, investiguen los materiales de enseñanza y entiendan la necesidad de abordar temas controvertidos en el aula.
Facultad de Educación, seguramente este rechazo disminuiría progresivamente en cada curso. Debe ser una prioridad en los programas de las Facultades de Educación formar a sus docentes para que sean capaces de generar espacios donde aquellas personas todavía en formación exploren sus creencias, investiguen los materiales de enseñanza y entiendan la necesidad de abordar temas controvertidos en el aula. Dichos objetivos no pueden ser exclusivos de sesiones de una sola clase o actividades cortas en un curso independiente. No se puede pasar por alto la necesidad de fomentar un entorno en el que maestras y maestros se reconozcan como aliados del colectivo LGTBQ en las aulas de las escuelas públicas. La meta radica en formar a las y los docentes del futuro con herramientas para enseñar que la diversidad nos enriquece a todas las personas favorece la vida del alumnado LGTBQ, en definitiva, contribuye a construir una escuela inclusiva, una escuela queer.

\section{Referencias}

Aguilar, C. (2008). Lectura, género, feminismo y LIJ. Lenguaje y Textos, 28, 113-128. 
Aguilar, C. (2017). LIJ y diversidad sexual LGTBI en la formación de maestros. CLIJ: Cuadernos de literatura infantil y juvenil, 30(276), 6-19.

Barozzi, S. (2016). Teacher training on gender and sexual identities in a Spanish context (Tesis doctoral inédita). Universidad de Granada, Granada, España. Recuperada de: https://hera.ugr.es/tesisugr/25961913.pdf

Blackburn, M. V., y Buckley, J. F. (2005). Teaching queer-inclusive English language arts. Journal of Adolescent \& Adult Literacy, 49(3), 202-212. doi: 10.1598/JAAL.49.3.4

Borrillo, D. (2001). Homofobia. Barcelona: Bellaterra.

Britzman, D. P. (1995). Is there a queer pedagogy? Or, stop reading straight. Educational theory, 45(2), 151-165. doi: 10.1111/j.1741-5446.1995.00151.x

Buchanan, L. B., Tschida, C., Bellows, E., \& Shear, S. B. (2019). Positioning children's literature to confront the persistent avoidance of LGBTQ topics among elementary preservice teachers. The Journal of Social Studies Research, 44(1), 169-184. doi: 10.1016/j.jssr.2019.01.006

Butler, J. (1994). Against Proper Objects. Differences: A Journal of Feminist Cultural Studies, (6), 1-26.

Carrera-Fernández, M.V., Cid-Fernández, X. M., Almeida, A., González-Fernández, A., y LameirasFernández, M. (2018). Actitudes hacia la diversidad cultural de adolescentes de secundaria españoles y portugueses: influencia de la heteronormatividad y la desconexión moral hacia el bullying. Revista de Psicodidáctica, (23), 17-25. doi: doi.org/10.1016/j.psicod.2017.07.004

Cohen, H. S., y Chasnoff, D. (1997). It's elementary: Talking about gay issues in school [Video]. San Francisco: Women's Educational Media. Recuperado de: https://www.youtube.com/watch?v=uMU9BCV05w\&list=PLL8ToG6BqAnEwLj8q-DA_W9YYSO7BwBmg

Cumming-Potvin, W. y Martino, W. (2014). Teaching about queer families: Surveillance, censorship, and the schooling of sexualities. Teaching Education, 25(3). 309-333. doi: $10.1080 / 10476210.2014 .889672$

De Haan, L., y Nijland, S. (2004). Rey y Rey. Barcelona: Serres, RBA.

Eyre, L. (1997). Compulsory heterosexuality in a university classroom. En M. Bryson y S. De Castell (Coord.), Radical in<ter>ventions: Identity, politics, and difference/s on educational praxis, (pp. 233-246). New York: Sunny Press.

Hermann-Wilmarth, J. M., \& Bills, P. (2010). Identity shifts: Queering teacher education research. Teacher Educator, 45(4), 257-272. doi:10.1080/08878730.2010.508324

Herthel, J., Jennings, J., y McNicholas, S. (2015). Soy Jazz. Barcelona: Bellaterra.

Jagose, A. (1996). Queer Theory. Melbuourne: Melbourne University Press.

Jennings, K. (Ed.). (1994). Becoming visible: A reader in gay \& lesbian history for high school \& college students. Los Angeles: Alyson Books.

Jennings, T., y Sherwin, G. (2008). Sexual orientation topics in elementary teacher preparation programs in the USA. Teaching Education, 19(4), 261-278. doi: 10.1080/10476210802436328

Killoran, I., y Pedleton Jiménez, K. (2007). "Unleashing the unpopular": Talking about sexual orientation and gender diversity in education. Olney, MD: Association for childhood Education International.

Kissen, R. M. (2002). Getting ready for Benjamin: Preparing teachers for sexual diversity in the classroom. Lanham MD: Rowman y Littlefield.

Leal, P., y Crookes, G. V. (2018). "Most of my students kept saying,'I never met a gay person'”: A queer English language teacher's agency for social justice. System, 79, 38-48. doi: 10.1016/j.system.2018.06.005 
Lipkin, A. (2002). The challenges of gay topics in teacher education: Politics, content, and pedagogy. En R. Kissen (Coord.), Getting ready for Benjamin: Preparing teachers for sexual diversity in the classroom (pp. 13-27). Lanham MD: Rowman and Littlefield.

Llorens, F., y Terol, S. (2015). Educación literaria, pensamiento crítico y conciencia ética: La composición, de Antonio Skármeta. América sin nombre, (20), 102-109. doi:10.14198/AMESN.2015.20.09

Lynch, L. (2000). Cruising the Library. En W. J. Spurlin (Coord.), Lesbian and Gay Studies and the Teaching of English: Positions, Pedagogies, and Cultural Politics (pp. 3-13). Urbana, IL: National Council of Teachers of English.

Martino, W. J., y Cumming-Potvin, W. (2011). “They Didn't Have Out There Gay Parents-They Just Looked Like Normal Regular Parents": Investigating Teachers' Approaches to Addressing SameSex Parenting and Non-normative Sexuality in the Elementary School Classroom. Curriculum Inquiry, 41(4), 480-501. doi:10.1111/j.1467-873X.2011.00557.x

Martino, W. J., y Cumming-Potvin, W. (2014). Teaching about "princess boys" or not: The case of one male elementary school teacher and the polemics of gender expression and embodiment. Men and Masculinities, 18(1), 79-99. doi:10.1177/1097184X14551278

Martino, W. J., y Cumming-Potvin, W. (2016) Teaching about sexual minorities and "princess boys": A queer and trans-infused approach to investigating LGBTQ-themed texts in the elementary school classroom. Discourse: Studies in the Cultural Politics of Education, 37(6), 807-827. doi: 10.1080/01596306.2014.940239

Mayo, C. (2013). LGBTQ youth and education: Policies and practices. New York: Teachers College Press.

Mendieta, M. J., y Piérola, M. (2006). Aitor tiene dos mamás. Barcelona: Bellaterra.

Meyer, E. J. (2015). The Personal Is Political: LGBTQ Education Research and Policy Since 1993. The Educational Forum, 79(4), 347-352. doi:10.1080/00131725.2015.1069514

Miller, H. M. (1999). Swimming with the Sharks. Reading Teacher, 52(6), 632-34.

Moje, E. B., y MuQaribu, M. (2003). Literacy and sexual identity. Journal of Adolescent \& Adult Literacy, 47(3), 204-208.

Nieto, S., y Bode, P. (2012). Affirming diversity: The sociopolitical context of multicultural education. Boston, MA: Pearson.

Oltra-Albiach, M. À., y Pardo-Coy, R. (2017). Gènere i diversitat sexual en la literatura infantil valenciana del segle XXI: una anàlisi dels materials literaris a l'aula d'educació infantil. Confluenze. Rivista di Studi Iberoamericani, 9(2), 28-40.

Oltra-Albiach, M. À., y Pardo-Coy, R. (2016). Educar en la diversitat. Una proposta d'educació literària en l'aula de Magisteri. Ítaca. Revista de Filologia. 2016, (7), 63-71.

Paiz, J. M. (2018). Queering ESL teaching: Pedagogical and materials creation issues. TESOL Journal, 9(2), 348-367. doi:10.1002/tesj.329

Paiz, J. M. (2019). Queering Practice: LGBTQ+ Diversity and Inclusion in English Language Teaching. Journal of Language, Identity \& Education, 18(4) 1-10. doi:10.1080/15348458.2019.1629933

Penna Tosso, M. (2012). Formación del profesorado en la atención a la diversidad afectivo-sexual (Tesis doctoral). Universidad Complutense de Madrid, Madrid. Recuperado de http://eprints.ucm.es/16718/1/T34011.pdf

Quintiá X., y Quarello, M. (2007). Titiritesa. Pontevedra: OQO editora.

Ramírez, M. (2012). La experiencia escolar en las narrativas de identidad sexual LGTB: un estudio fenomenológico retrospectivo (Tesis doctoral inédita). Universidad Autónoma de Madrid, Madrid. 
Renovell Rico, S. (2017). Actitudes del alumnado de educación secundaria hacia la diversidad afectivo-sexual y familiar. Estudio descriptivo en las cooperativas valencianas y propuesta de intervención. (Tesis doctoral). Universitat de València, València. Recuperada de http://roderic.uv.es/bitstream/handle/10550/61343/Tesis\%20y\%20Programa\%20HEDUCA.pdf?sequence=3yisAllowed $=\mathrm{y}$

Sánchez Sáinz, M. (2019). Pedagogías Queer: ¿nos arriesgamos a hacer otra educación?, Madrid: La Catarata.

Sánchez-Torrejón, B. (2017). La diversidad afectivo-sexual desde la mirada de la comunidad educativa de la etapa de educación primaria: Un estudio cualitativo (Tesis doctoral inédita). Universidad de Cádiz, Cádiz, España.

Schall, J., y Kauffmann, G. (2003). Exploring Literature with Gay and Lesbian Characters in the Elementary School. Journal of Children's Literature, 29(1), 36-45.

Sears J. T. (1993). Challenges for educators: Lesbian, gay, and bisexual families. The High School Journal, 77(1/2), 138-156.

Spurlin, W. J. (2000). Lesbian and Gay Studies and the Teaching of English: Positions, Pedagogies, and Cultural Politics. Urbana, IL: National Council of Teachers of English.

Straut, D., y Sapon-Shevin, M. (2002). "But no one in the class is gay": Countering invisibility and creating allies in teacher education programs. En R. Kissen (Coord.), Getting ready for Benjamin: Preparing teachers for sexual diversity in the classroom (pp. 29-41). Lanham MD: Rowman and Littlefield.

Sumara, D., y Davis, B. (1998). Telling tales of surprise. En W.F. Pinar (Coord.), Queer theory in education (pp. 197-219). New York: Routledge.

Soler-Quílez, G. (2019). Personajes trans en la tradición popular castellana: introduciendo el arcoíris en el aula. En M.M. Campos y M. C. Quiles (Coord.), Repensando la didáctica de la lengua y la literatura. Paradigmas y líneas emergentes de investigación, (pp. 297-305). Madrid: Visor.

Steck, A., y Perry, D. (2017). Secondary school leader perceptions about the inclusion of queer materials in the school course curricula. The Curriculum Journal, 28(3), 327-348. doi: 10.1080/09585176.2017.1292180

Swartz, P. C. (2003). It's elementary in Appalachia: Helping prospective teachers and their students understand sexuality and gender. Journal of Gay \& Lesbian Issues in Education, 1(1), 51-71.

Temple, C. A., Martinez, M., Yokota, J., y Naylor, A. (2006). Children's books in children's hands: An introduction to their literature. Boston: Pearson Allyn and Bacon.

\section{How to cite this paper:}

Soler-Quílez, G., (2020). Queerizando las Facultades de Educación: una escuela inclusiva es posible. Journal of Literary Education, (3), pp. 26-43. doi: 10.7203/JLE.3.16699 